\title{
ASSESSMENT OF THE STATE OF EMOTIONAL HEALTH OF UKRAINIAN UNIVERSITY STUDENT YOUTH THAT IS INVOLVED INTO DIFFERENT LEVELS OF PHYSICAL ACTIVITY
}

\author{
Marta Yaroshyk \\ Lviv State University of Physical Culture, Ukraine \\ Halyna Malanchyk \\ Lviv State University of Physical Culture, Ukraine \\ Alla Solovei \\ Lviv State University of Physical Culture, Ukraine
}

\begin{abstract}
In the present days, we encounter and can observe a widespread distribution of physical, moral and psychological crisis among the citizens of Ukraine. The most inclined to undergo and experience distress group of the population is higher educational institutions' youth. Based on the data we have collected and carefully analyzed, we have discovered that regular exposure of a person to physical activity helps maintain the levels of distress and influence of other negatively oriented emotional states to their minimums whereas the physical activity positively impacts emotional health, especially in regard to student youth. Accordingly, our research is dedicated and conducted to investigate and assess how emotional health of Ukrainian students correlates with different levels of physical activity they experience. In order to complete the study, we asked a few female students that indeed take physical training (PT) classes at their universities, precisely, 48, to take part in our investigation. The participants were at the particular stages of their Bachelor's program progression (from Freshmen to Senior Year students) at the moment of the study being conducted. We attempt to measure and assess the indices that constitute emotional health which we defined as a) mood, b) well-being, c) overall activity, and d) anxiety. Additionally, we determine the levels of physical activity of each student. Based on the evidence we have collected, the students evaluated their moods in the range of 56 $\pm 10,0$ points (high evaluation marks), their well-beings in the range of $51 \pm 10,7$ points (high evaluation marks), and the levels of their overall activity in the range of 43 \pm 9,5 points (average evaluation marks). Altogether, the vast majority of the participants marked their levels of trait and state anxieties

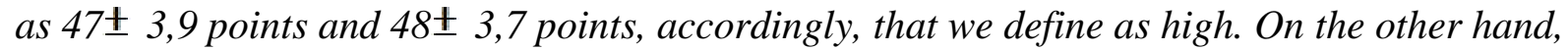
we have discovered that most participants have low physical activity index that constitute into the value of 31. On the contrary, the ones whose physical activity is higher have stated that their state anxiety is lower - 46,3 $\pm 3,4$ points, - compared to those who involve into average and low exposure to physical activity - 47,2 \pm 3,5 points and 47,6 \pm 4,3 points, respectively. The potential of future studies of emotional health are highly dependent upon researches into emotions that students experience, how well students recognize, distinguish,
\end{abstract}


Yaroshyk et al., 2020. Assessment of the State of Emotional Health of Ukrainian University Student Youth that is Involved into Different Levels of Physical Activity

and live through their feelings, as well as into the abilities of students to adapt to alternate vital circumstances.

Keywords: emotional health, physical activity, students.

\section{Introduction}

We have noticed the crisis of physical, moral and psychological health of population nowadays in Ukraine. The vulnerable part of population are students (Vasilievih, 2010; Skorbach \& Shastun, 2013). Students' life is in conditions of nervous tension and permanent stresses. Numerous researches determined positive correlation between motor activity and students' emotional health (Penedo \& Dahn, 2005; Strong et al., 2005; Biddle \& Asare, 2011; Ahn \& Fedewa, 2011).

Few scientists have investigated the problem of students' emotional health in Ukraine. Only some aspects of this problem are clarified. The influence of emotional health on harmonious development of students as person has been described by L.O. Kolisnyk (2010). The author states that numerous amount of students have deviation in emotional sphere. T.V. Scorbach and T.O. Shastun found out socio-psychological factors of students' physical health infringement (Skorbach \& Shastun, 2013). Their research indicates that first year students and students from countryside are in the risk groups. A lot of investigations are about emotional burnout of students (Maslach \& Leiter, 1997; Pomitkina \& Kovalkova, 2008; Lipson, Lattie, \& Eisenberg, 2019). Within, some research determined methods of improving students' psychological health (Vasilievih, 2010; Breus, 2013; Zavydivska, Rymar, \& Malanchuk, 2015). The popular methods are motor activity optimization, emotional intelligence development, positive motivation and settings creating.

Even though that correlation between motor activity and emotional health is obvious, the discussion about its meaning are still exist (Strong et al., 2005). Perhaps, regular physical trainings improve emotional health or people are more physically active when they are healthy psychologically. That is why, we have decided to made evaluation of indicators of emotional health of students with different motor activity and take part in discuss the question.

\section{Methods}

We have applied such methods as theoretical analysis, generalization of informational sources and questionnaire for solving the goal of research. 48 students from 1-2 years of studying that regularly go for sport and attend physical education classes have been taken part in the research. We have provided the evaluation of such indicators of emotional health of students as 
well-being, activity, mood and level of anxiety. According to the goal we provide the students' questionnaire by two surveys: self-being-activity-mood (Doskin, Lavrent'eva, Miroshnikov \& Sharaj, 1973) and Spilberg-Hanin (Greben', 2007). While processing data according the survey well-beingactivity-mood we have evaluated each indicator in the principle: $<30$ points low rate, 30-50 points - average rate, $>50$ points - high rate. In the result of second survey we have established levels of individual and reactive anxiety of students. Within, the point below 30 represents low level of anxiety, the point between 31 and 45 represent average level of anxiety and point, higher that 45 testifies the high level of anxiety.

The evaluation of motor activity we have made according the methodic from Framingham research (Davidenko \& Masaud, 1999). We have analyzed students' daily expenses of time for various types of activity and determined the index of motor activity. In order to determine the quantitative value (in points) for different types of motor activity (according its intensity) we have applied weighting coefficients of motor activity (Table 1). Thus, the time (in hours), devoted for some level of motor activity is multiplied by appropriate coefficient.

Table 1 Physical Activity Mass Coefficient (Kannel \& Sorlie, 1979)

\begin{tabular}{|l|c|c|}
\hline \multicolumn{1}{|c|}{$\begin{array}{c}\text { Level of Physical } \\
\text { Activity }\end{array}$} & $\begin{array}{c}\text { Oxygen Consumption, } \\
\text { liters/minute }\end{array}$ & Mass coefficient \\
\hline Basic & 0.25 & 1.0 \\
\hline Time Spent Sitting & 0.28 & 1.1 \\
\hline Low & 0.41 & 1.5 \\
\hline Average & 0.60 & 2.4 \\
\hline High & 1.25 & 5.0 \\
\hline
\end{tabular}

The evaluation of motor activity we provide according the scale: $<31$ points - low index; 31-34 points - average index; >34 - high index.

In the process of statistical processing of results of the research we have calculated such statistical characteristics: arithmetic mean $\bar{x}$ and mean square deviation $\sigma$.

\section{Results}

The prime indicators of students' emotional health that we have evaluated and assessed during our study are as enlisted: 1) well-being, 2) overall activity, 3) mood, and 4) anxiety levels. Our investigation provides the following 
Yaroshyk et al., 2020. Assessment of the State of Emotional Health of Ukrainian University Student Youth that is Involved into Different Levels of Physical Activity

evidences: generally, the participants marked their well-being levels as $51 \pm 10.7$ points, which we consider as a high level of the measure (Fig. 1).

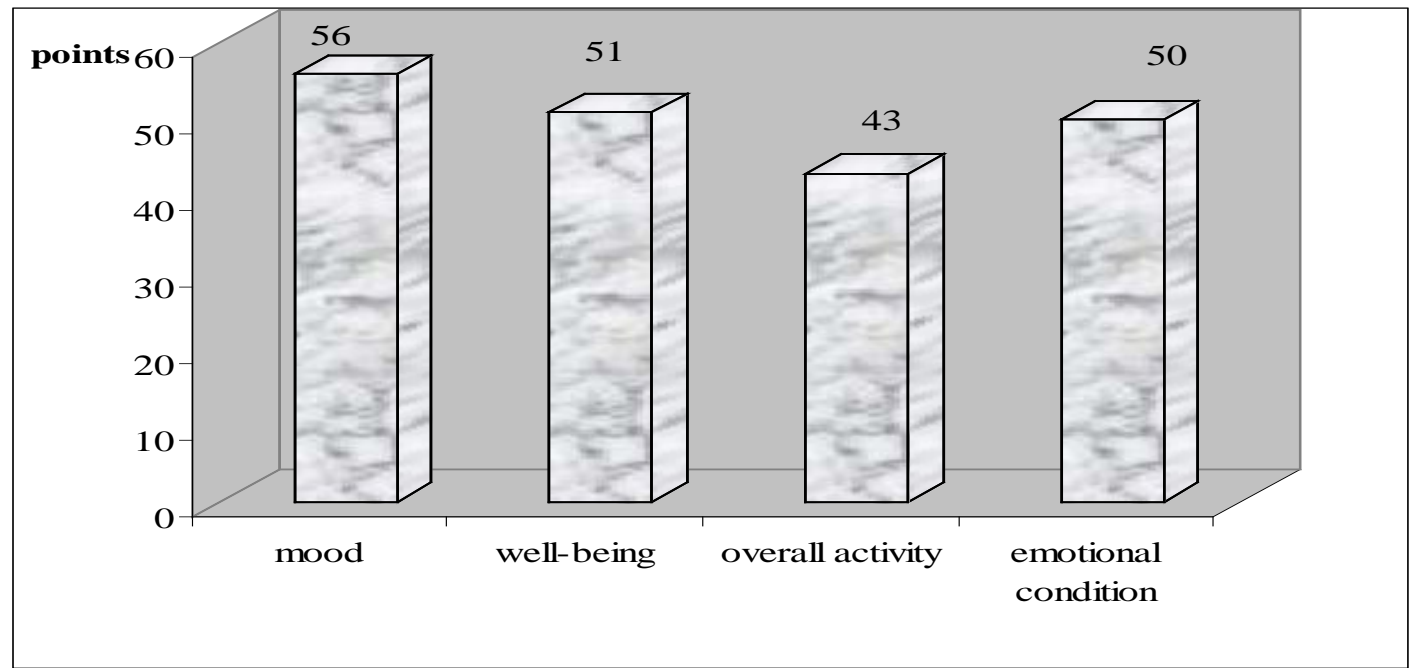

Note. The principles of emotional condition evaluating is made according the methodic of well-being-activity-mood: $<30$ points - low rate; $30-50$ points - average rate; $>50$ points high rate

\section{Figure 1 Students' self-esteem of emotional condition}

Based on that evidence, we arguably state that the participants' well-being is high enough to actively and without any restrictions educate themselves within the facilities of the higher educational institution. According to the conducted research results, we have determined that the lowest level of motional activity of the participants is in the range of $43 \pm 9.5$ points (average level). Henceforth, the vast majority of the ones who took part in the study was active, mobile, fast, able to perform at a decent level of any endeavor, excited, and patient enough. Nonetheless, a few students stated that they were inclined to regularly be sleepy during the work days, and that they would like to be resting more often.

One of the most notable aspects of the study is that the students highly evaluated their mood $-56 \pm 10.0$ points, which, in turn, is the most highlyevaluated indicator amongst the emotional health indicators. Nevertheless some positive responses regarding their moods, we still have to pay attention to the fact that student's cycle incorporates high levels of excitement at the beginning of the degree acquisition process to high levels of skepticism when it comes to assessment of higher educational institutions' regimes alongside the methodologies of pedagogy and of some particular pedagogues.

$62 \%$ of the participants have declared that they experience high levels of trait anxiety, resulting into $50 \pm 3.1$ points of the measure (Table 2). The 
remainder $38 \%$ have scored $44 \pm 1.5$ points having average levels of trait anxiety. These observations can lead us to a conclusion that the students appear at a vitally important turning points of their lives where they have to choose carefully what to do for living and how to proceed further in life, therefore, arriving at being stressed about their futures, both about everyday activities and the closest circles of each of them. The respondents adequately react to stressful situations, and the levels of their both trait and state anxieties correspond to the norms, which, in turn, entails no further special treatment of the participant audience group.

Table 2 The evaluation of individual and reactive students' anxiety (n=48)

\begin{tabular}{|c|c|c|c|c|}
\hline \multirow{2}{*}{ Indicators } & \multicolumn{2}{|c|}{ Individual anxiety } & \multicolumn{2}{c|}{ Reactive anxiety } \\
\cline { 2 - 5 } & Rate, points $(\mathrm{x} \pm \sigma)$ & $\%$ & Rate, points $(\mathrm{x} \pm \sigma)$ & $\%$ \\
\hline high level of anxiety & $50 \pm 3.1$ & 62 & $49 \pm 2.6$ & 73 \\
\hline $\begin{array}{c}\text { average level of } \\
\text { anxiety }\end{array}$ & $44 \pm 1.5$ & 38 & $43 \pm 2,0$ & 27 \\
\hline arithmetic mean & \multicolumn{2}{|c|}{$47 \pm 3.9$} & \multicolumn{2}{c|}{$48 \pm 3.7$} \\
\hline
\end{tabular}

Thus, accordingly to the results of the investigation, on average, the female students' trait anxiety levels are high, having $47 \pm 3.9$ points. We conclude that such levels of trait anxiety are characteristic of the participants' age group. On the other hand, the state anxiety levels indicate high marks as well, resulting into $49 \pm 2.6$ points for $73 \%$ of those who took part in the study. Two most widespread features of such students are closeness and decreased levels of social activity and involvement. Primarily, this group of people share the trait of being non-initiative as well as their expectations for lives are severely lowered compared to the one whose state anxiety levels are closer to the standards. Too, they have lower self-esteem and expectant to fail more often, therefore, becoming less ambitious. The rest $27 \%$ scores for $43 \pm 2.0$ points that lies within the boundaries of the norm. It translates into moderate level of tranquility among the group representatives, they are more active and friendly; although, some of them seldom experience anxiety that does not regard the circumstances at hand.

Based on the date we collected by exposing the participants to fill in the questionnaire, we can infer that, on average, the students tend to have high level of state anxiety - 48 \pm 3.7 points. The increased levels of state anxiety feature subjectively deformed well-being comprehension of oneself. These females have a higher tendency to anticipate and perceive threats when there are none, with the respect to their self-esteem and overall security in a wide variety of life circumstances, reacting over-stressfully with apparent behavioral changes and further complications. Unfortunately, throughout the process of the research 
Yaroshyk et al., 2020. Assessment of the State of Emotional Health of Ukrainian University Student Youth that is Involved into Different Levels of Physical Activity

conduction, we have not identified any participant whose state anxiety level is below the averages. It allows us to draw a conclusion, that the majority of the students experience disbelief, lack of confidence, and worry, especially when they encounter extremely discomfort situations. Subsequently, a regular exposure to such circumstances could lead to neurotic disorders, infarcts, or preinfarction states.

In order to determine the levels of motional activity of the participants, we utilized the Framingham methodologies. Surprisingly, we have collected enough data to conclude that the corresponding levels are low - 31 point of the measurement scale. Considering the fact that the Framingham methodologies' indicators have to display at the very least 42 to be deemed optimal, none of the respondents who took part in the investigation has such a result. Based on these data, we affirm that students are involved into physical activity too rarely. The distribution of time spent in a specific way is 33\% for time spent sitting, 30\% for basic motional activity, and 29\% for low motional activity level (Fig. 2). On the contrary, during the weekends, the distribution is as following - $36 \%$ of the time is dedicate to basic motional activity, $39 \%$ for sitting, and $14 \%$ for the average level of motional activity.

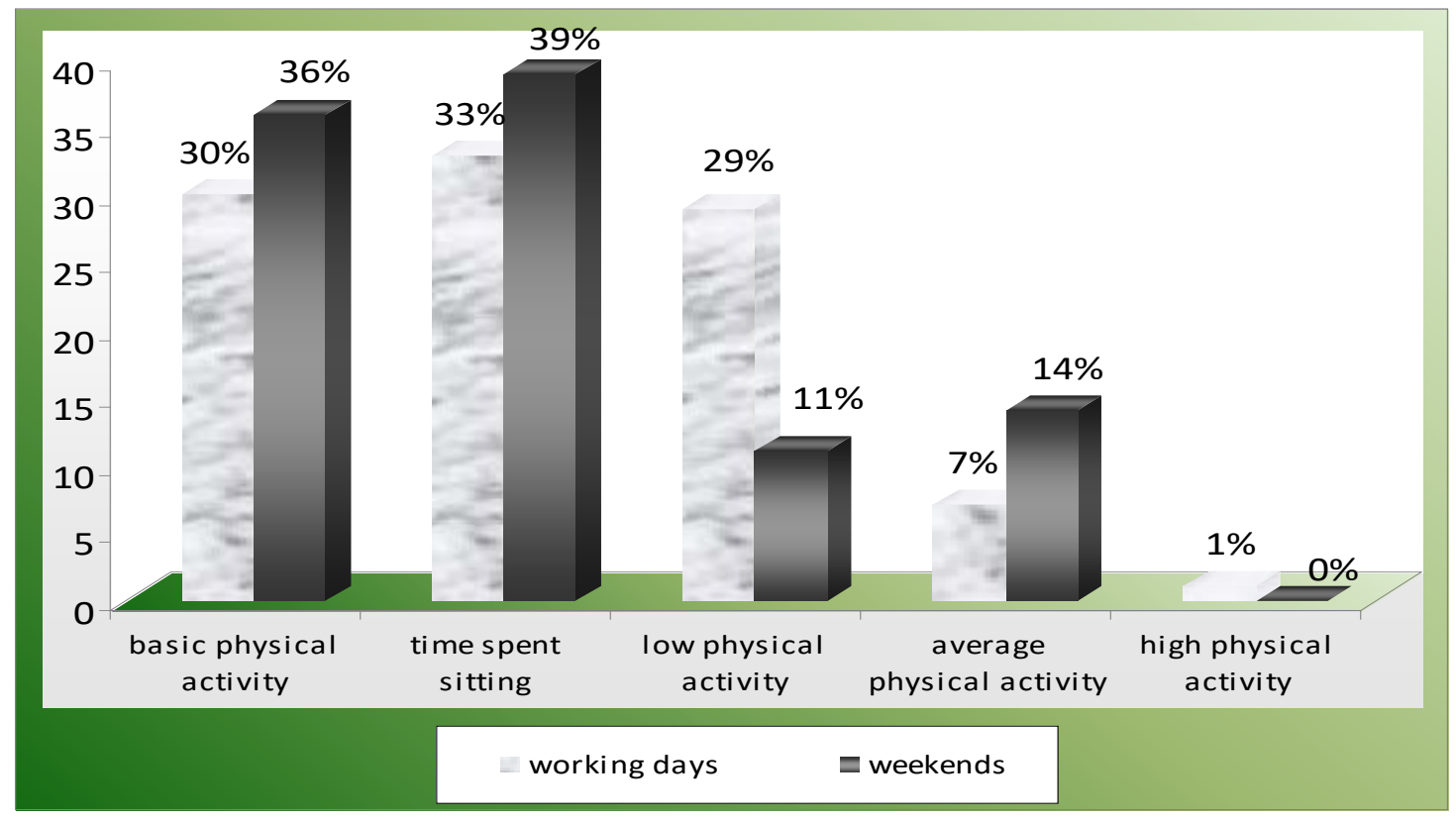

Figure 2 Students' distribution by levels of motor activity

Having thoroughly analyzed the data we computed with the regard to the indicators of emotional health, considering that the participants will be associated with a particular level of involvement into physical activities, the results are the following with the respect to the "mood" indicator — the highest 
score of $55.2 \pm 10.8$ points (high evaluation) represents the group of students whose physical activity index is average (Table 3). The representatives of the low physical activity index score have $55 \pm 9.6$ points (high evaluation) against the measurement scale, whereas those who are highly involved into physical activity evaluated their mood as $54.7 \pm 11.4$ (high evaluation), which by surprise is the lowest among all of the groups.

Table 3 The evaluation of indicators of students' emotional health with different motor activity (n=48)

\begin{tabular}{|c|c|c|c|}
\hline \multirow{2}{*}{ Indicators } & \multicolumn{3}{|c|}{ Index of physical activity (IPA) } \\
\cline { 2 - 4 } & High & Average & Low \\
\hline Well-being & $49.9 \pm 0.3$ & $51.3 \pm 11.2$ & $48.3 \pm 10.1$ \\
\hline Activity & $45.3 \pm 10.6$ & $44.7 \pm 9.4$ & $39.7 \pm 7.6$ \\
\hline Mood & $54.7 \pm 11.4$ & $55.2 \pm 10.8$ & $55.0 \pm 9.6$ \\
\hline Individual anxiety & $46.7 \pm 5.6$ & $47.6 \pm 4.4$ & $46.4 \pm 3.6$ \\
\hline Reactive anxiety & $46.3 \pm 3.4$ & $47.2 \pm 3.5$ & $47.6 \pm 4.3$ \\
\hline
\end{tabular}

Note. The principles of evaluation by the methodic well-being-activity-mood:

$<30$ points - low rate; 30-50 points - average rate; $>50$ points - high rate.

Principles of evaluation anxiety levels by methodic Spilberg-Hanin:

$<30$ points - low anxiety level; 31-45 points - average anxiety level; $>45$ high anxiety level.

Comparing to the physical activity indexes of each group, we can infer that the participants objectively assessed and identified their overall activity, that is the other emotional health indicator. The lowest mark has been hit by the group of students whose index is low, corresponding to $39.7 \pm 7.6$ points. The highest score of $45.3 \pm 10.6$ points is representative of the high physical activity index group. $44.7 \pm 9.4$ points is the result of the average group.

Speaking of the well-being indicators, the score vary as well. The best state of well-being is characteristic of the group with the average index $-51.3 \pm 11.2$ points. $49.9 \pm 10.3$ points and $48.3 \pm 10.1$ points are respectively representative of the high and low indices of the physical activity.

Drawing an overall conclusion, we can state that students decreasingly involve into physical activities that leads to depreciation of the overall organism functionality, thus, the students become less trained and enduring. To become trained and overall perform better, it is necessary for one to experience highintensity physical activities, for instance, well organized and planned intensive training sessions. Taking to the consideration the fact that such trainings are a part of high-intensity level of physical activity, most students do not possess such a listing on their everyday schedule.

As well, we have discovered that physical activity influence anxiety levels. Thus, the students whose physical activity index is high score in $46.3 \pm 3.4$ points against the state anxiety measurement scale. $47.2 \pm 3.5$ points and $47.6 \pm 4.3$ points 
Yaroshyk et al., 2020. Assessment of the State of Emotional Health of Ukrainian University Student Youth that is Involved into Different Levels of Physical Activity

are representative of those who have average and low indices, respectively. The lower the index, the higher level of state anxiety is expected. Hence, the students with the lowest scores tend to react more adequately to the most significant socio-psychological distress sources (expectancies of negative judgements by other people, expectancies of aggressive response by other people, acceptance of inappropriate treatment of them by others, threats to self-esteem and selfrespect, the prestige level), which, in turn, leads to both better states of physical and emotional health.

\section{Conclusions and discussion}

The elaborated research represented high level of well-being, mood and activity of students that took part in the research. Within, the indicators from research did not change significantly. The highest rate students gave to their mood. However, we were concerned about level of students' anxiety. We noticed high level of reactive anxiety in $73 \%$ of students. The higher level of anxiety can be the subjective display of personality problems. It is difficult to work with such students. They are reserved, low sociable, have low motivation and show more behavioral and discipline problems. As a rule, they are uninitiated, that is connected with expectation of fails and low confidence in themselves. However, students with high motor activity got lower level of anxiety, than students with average and low activity levels. Data, we have got are confirm researches of lots of authors (Strong et al., 2005; Penedo \& Dahn, 2005; Biddle \& Asare, M., 2011) that physical load decrease symptoms of depression and anxiety and improve their mood.

The research we have made represented now index of students' physical activity according Framingham methodic. The data we got is not respond declared students' self-esteem according methodic well-being-activity-mood. It may indicate about lack of students' knowledge about norms of motor activity that is appropriate to their age. Accordingly, their motivation is formed in due to support proper motor activity. We suppose that increasing of volume of motor activity would minimize anxiety among students. Within, the research of Penedo and Dahn (Penedo \& Dahn, 2005) represent that regular physical load may prevent symptoms of depression and axiety.

There is much potential for further studies aimed at finding correlation between emotional health and physical activity. They will primarily depend upon investigations of students' emotions, their abilities to recognize, distinguish and live through their feelings, as well as of the abilities of youth to adapt to alternate vital circumstances. 


\section{References}

Ahn, S., \& Fedewa, A. (2011). A meta-analysis of the relationship between children's physical activity and mental health. Journal of Pediatric Psychology, 36(4), 385-397.

Biddle, S., \& Asare, M. (2011). Physical activity and mental health in children and adolescents: A review of reviews. British Journal of Sports Medicine, 45(11), 886-895.

Breus, J. (2013). The impact of emotional intelligence development on keeping personal emotional health. The research of young scientists in the context of modern science development: issues from III all-Ukrainian scientific-practical conference, 1, 162-167.

Davidenko, E., \& Masaud, R. (1999). The methodic of Framingham research of motor activity of people: recommendations for applying. Kyiv: Olympic literature.

Doskin, V., Lavrent'eva, N., Miroshnikov, M., \& Sharaj, V. (1973). The test of differentiated self-esteem of functional condition. The questions of psychology, 6, 141-145.

Greben', N. (2007). Psychological tests for professionals. Minsk: modern school.

Kannel, W., \& Sorlie P. (1979). Some health benefits of physical activity. The Framingham Study. Arch Intern Med, 139(8), 857-861.

Kolisnyk, L. (2010). The emotional health influences on harmonious development of a student's individuality. All-Ukrainian scientific-practical conference with international participation "Education and health: formation of health of children, adolescents and young people in the conditions of an educational institution”, 1, 71-80.

Lipson, S., Lattie, E., \& Eisenberg, D. (2019). Increased rates of mental health service utilization by U.S. college students: 10-year population-level trends (2007-2017). Psychiatric Services, 70(1), 60-63.DOI: 10.1176/appi.ps.201800332

Maslach, C., \& Leiter, P. (1997). The Truth About Burnout: How Organizations Cause Personal Stress and What to Do About It. San Francisco: Jossey-Bass Publishers.

Penedo, F., \& Dahn, J. (2005). Exercise and well-being: A review of mental and physical health benefits associated with physical activity. Current Opinion in Psychiatry, 18(2),189-193.

Pomitkina, L., \& Koval'kova, T. (2008). The problem of emotional burnout of students. Social psychology: Ukrainian scientific journal, 6, 94-101.

Skorbach, T., \& Shastun, T. (2013). The socio-psychological factors of psychological health deviations of student youth. The relevant issues and approaches to improve the process of nurture at educational institutions, 1, 5-7.

Strong, W., Malina, R., Blimkie, C., Daniels, S., Dishman, R., Gutin, B., Hergenroeder, A., Must, A., Nixon, P., Pivarnik, J., Rowland, T., Trost, S., \& Trudeau, F. (2005). Evidence based physical activity for school-age youth. Journal of Pediatrics, 146(6), 732-737.

Vasilievih, L. (2010). The impact of studying and examination stress on the process of adaptation of first year students in higher educational institution. Collection of scintific works of Khmelnytskyy institution of social technologies of University "Ukraine", 2, 226-228.

Zavydivska, N., Rymar, O., \& Malanchuk, H. (2015). Innovative mechanisms of improvement of student's physical education system. Journal of Physical Education and Sport, 15(4), 758-764. 



\section{PĒTĪJUMI EKONOMIKAS UN VADĪBAS JOMĀ ILGTSPĒJĪGAI IZGLİTĪBAI \\ Researches in Economics and \\ Management for Sustainable Education}


